\title{
Correlating gastric emptying of amphotericin B and paracetamol solid lipid nanoparticles with changes in particle surface chemistry
}

\author{
Hilda Amekyeh ${ }^{\mathrm{a}}$, Nashiru Billa ${ }^{\mathrm{a}, *}$, Clive Roberts $^{\mathrm{b}}$
}

a School of Pharmacy, Faculty of Science, University of Nottingham Malaysia Campus, Jalan Broga, 43500 Semenyih, Selangor Darul Ehsan, Malaysia

b School of Pharmacy, University of Nottingham, University Park, Nottingham NG7 2RD, United Kingdom

"Corresponding Author:

Email : Nashiru.Billa@nottingham.edu.my

Tel : $\quad+60389248211$

Fax : +60389248018 


\section{ABSTRACT}

Oral delivery of pharmaceuticals requires that they retain their physical and chemical attributes during transit within the gastrointestinal (GI) tract, for the manifestation of desired therapeutic profiles. Solid lipid nanoparticles (SLNs) are used as carriers to improve the absorption of

5 hydrophobic drugs. In this study, we examine the stability of amphotericin B (AmB) and paracetamol (PAR) SLNs in simulated GI fluids during gastric emptying. On contact with the simulated fluids, the particles increased in size due to ingress of the dissolution media into the particles. Simulated gastric emptying revealed that the formulations had mean sizes $<350 \mathrm{~nm}$ and neutral surface charges, both of which are optimal for intestinal absorption of SLNs. There was ingress of the fluids into the SLNs, followed by diffusion of the dissolved drug, whose rate depended on the solubility of the loaded-drug in the particular medium. Time-of-flight secondary ion mass spectrometry analyses indicated that drug loading followed the core-shell model and that the AmB SLNs have a more drug-enriched core than the PAR SLNs do. The AmB SLNs are therefore a very suitable carrier of AmB for oral delivery. The stability of the SLNs in the

15 simulated GI media indicates their suitability for oral delivery.

Keywords: Solid lipid nanoparticle, stability, gastrointestinal tract, gastric emptying, amphotericin B, paracetamol

Chemical compounds studied in this article Amphotericin B (PubChem CID: 5386092); Paracetamol (PubChem CID: 1983); Sodium cholate (PubChem CID: 23668194)

AmB, amphotericin B; DLS, dynamic light scattering; GI, gastrointestinal; NTA, nanoparticle tracking analysis; PAR, paracetamol; PDI, polydispersity index; SGF, simulated gastric fluid; SIF, simulated intestinal fluid; SLN, solid lipid nanoparticle; ToF-SIMS, time-of-flight secondary ion mass spectrometry; ZP, zeta potential. 


\section{Introduction}

Ascertaining the stability of formulations in biological media can provide critical information for optimising such preparations, particularly for submicron formulations. Stability studies conducted in appropriate simulated GI media may give information on the fate of formulations after their oral administration. The stability of nanoparticulate formulations may be ascertained with reference to their chemical or pharmaceutical/storage stabilities (Muthu and Feng, 2009).

After oral administration, a dosage form or drug must initially overcome the physical and chemical hydrodynamics within the GI tract and traverse the intestinal epithelium effectively in order to register significant bioavailability. Lipid-based delivery systems are susceptible to digestion/degradation by gastric and intestinal enzymes prior to their absorption. They can be absorbed by enterocytes as micelles, which improves the absorption of hydrophobic drugs (Müller et al., 1996; Pouton, 2000; Roger et al., 2009; Subramanian and Ghosal, 2004). In addition, solid lipid nanoparticles (SLNs) have a large surface area and are therefore prone to aggregation, which may reduce particle interaction with the intestinal mucosa (Jani et al., 1990).

The slow drug release characteristics exhibited by SLN systems assure evasion of GI degradation of susceptible drugs; however, this may depend on the rate of degradation of the particles, which is also governed by the type of excipients used in formulating the SLNs (Damgé et al., 1990; Olbrich et al., 2002a, 2002b). A study by Zhang et al. revealed that using SLNs averted the gastric irritation caused by triptolide, which indicates that the delivery system retains its integrity in the stomach; however, the composition of the lipid matrix varies among formulations hence each SLN may respond differently (Zhang et al., 2013). 
Amphotericin B $(\mathrm{AmB})$ is a polyene antibiotic with broad-spectrum antifungal activity and an anti-leishmanial activity. It is used to treat systemic mycoses and it is particularly useful in immunocompromised patients as there is a high mortality rate in such patients due to invasive fungal infections (Wasan et al., 2009). AmB is currently administered intravenously due its poor aqueous solubility characteristics, which makes oral delivery challenging. In earlier studies, we have successfully formulated and exhaustively characterised an oral AmB-containing solid lipid nanoformulation with the aim of improving its oral bioavailability (Amekyeh et al., 2015; Tan et

50 al., 2014; Tan et al., 2010).

In the following, we investigate the stability of the AmB SLNs when challenged by simulated GI media. This was done in comparison with an identical SLN formulation containing paracetamol (PAR), which was used for studying the gastric empty pattern of the AmB SLNs. These SLNs have been characterised previously with regard to bioavailability and GI transit

55 properties (Amekyeh et al., 2015). We aimed to specifically assess the changes in the surface chemistry and physical properties of the SLNs in simulated GI media and then extrapolate the data to the bioavailability study carried out earlier.

\section{Materials and methods}

\subsection{Materials}

Paracetamol, simulated gastric fluid (SGF) and simulated intestinal fluid (SIF) were purchased from Sigma-Aldrich (St. Louis, Missouri, USA). SGF and SIF were diluted with ultrapure water to obtain the media at $\mathrm{pH} 1.2$ and $\mathrm{pH} 6.6$ respectively. Beeswax was obtained from Acros Organics (New Jersey, USA), theobroma oil from JB Cocoa Company Limited 
(Johor, Malaysia) and amphotericin B from Nacalai Tesque Inc. (Kyoto, Japan). Lecithin soy and sodium cholate were obtained from MP Biomedicals (Illkirch, France). Chloroform, ethyl acetate and methanol were purchased from Fisher Scientific (Loughborough, UK). All the reagents and solvents used were of analytical and HPLC grades respectively. Ultrapure water $(18.2 \mathrm{M} \Omega \cdot \mathrm{cm}$ at $25^{\circ} \mathrm{C}$ ) was obtained using a Milli-Q system (Millipore Corp., Bedford, USA).

\subsection{Preparation of drug-free, AmB and PAR SLN formulations}

The drug-loaded SLNs were prepared according to the emulsification solvent diffusion technique, and characterised as described in our previous work (Amekyeh et al., 2015). Briefly, $50 \mathrm{mg}$ of drug, $120 \mathrm{mg}$ of lecithin and $200 \mathrm{mg}$ each of cocoa butter and beeswax were added to a 40-mL mixture of chloroform and methanol (1:1). The solvent was evaporated off using a Rotavapor $^{\circledR}$ R-200/205 (Büchi, Switzerland) at $50^{\circ}$ C. The resulting drug-lipid matrix was melted in $20 \mathrm{~mL}$ of ethyl acetate at $70^{\circ} \mathrm{C}$ and added to $40 \mathrm{~mL}$ of $2.5 \% \mathrm{w} / \mathrm{v}$ sodium cholate solution at $70^{\circ} \mathrm{C}$. The mixture homogenised (Ika-Turrax T $25^{\circledR}, \mathrm{IKA}^{\circledR}$, Staufen im Breisgau, Germany) at 10 $000 \mathrm{rpm}$ for $6 \mathrm{~min}$ to prepare an emulsion. About $60 \mathrm{~mL}$ of water at $70^{\circ} \mathrm{C}$ was then added slowly to the mixture with continuous stirring for $20 \mathrm{~min}$, after which the organic solvent was evaporated off. The drug-free formulation was similarly prepared without any drug.

\subsection{Stability of the SLNs in simulated GI fluids}

The SLN formulations were evaluated for their stability in SGF and SIF. An aliquot of 1 $85 \mathrm{~mL}$ of each formulation was placed in separate tubes containing SGF-only and SIF-only media, to produce a 1 in 250 dilution, which were then incubated for $2 \mathrm{hr}$. In a parallel study, the 
formulations were incubated in SGF for $2 \mathrm{hr}$, followed by transfer into SIF and incubation for an additional $2 \mathrm{hr}$ to mimic the gastric emptying process (SGF+SIF). All the SLN samples were diluted with the appropriate media to obtain a final dilution of 1 in 500 prior to analyses. The samples were then evaluated for size and surface charge changes using dynamic light scattering (DLS) and nanoparticle tracking analysis (NTA). Time-of-flight secondary ion mass spectrometry (ToF-SIMS) was used to qualitatively evaluate changes in the surface chemistries of the particles after their exposure to the media.

\subsection{DLS studies}

DLS studies on the SLNs were carried out using a Zetasizer Nano ZS ${ }^{\circledR}$ (Malvern, UK) and the parameters measured were z-average diameter, zeta potential (ZP) and polydispersity index (PDI). Each analysis was carried out at $37^{\circ} \mathrm{C}$ and performed in triplicate. The data obtained are expressed as mean \pm standard deviation (SD).

\subsection{NTA studies}

NTA measurements were performed using a NanoSight LM10 (NanoSight, Amesbury, United Kingdom) equipped with a sample chamber and a 640-nm laser. Using sterile syringes, samples were injected into the chamber to capacity. All measurements were done at $37^{\circ} \mathrm{C}$. Each video was captured at 30 frames/sec for $1 \mathrm{~min} 30 \mathrm{sec}$ and analysed using the NTA software version 3.1 Build 3.1.46. Triplicate measurements were performed for each sample and the data obtained have been expressed as mean \pm SD. 


\subsection{ToF-SIMS analyses}

ToF-SIMS was carried out on the samples using an ION-TOF equipment (Münster,

110 Germany) equipped with a liquid metal bismuth ion gun (LMIG). Prior to analyses, the SLNs were washed by centrifuging at $13000 \mathrm{rpm}$ for $60 \mathrm{~min}$, after which the supernatants were discarded and deionised water added to the samples. The washing was done to remove ions from the simulated GI media off the particles, as these ions can affect signals in the spectra. The washing also helped to remove traces of unassociated drug during formulation. The precipitated

115 particles were resuspended in water by shaking and the centrifugation process was repeated thrice. The washed SLNs were redispersed in deionised water and about $40 \mu \mathrm{L}$ samples placed on labelled silicon wafers. The samples were then air-dried prior to analyses. The analyses were carried out using $25 \mathrm{keV} \mathrm{Bi}_{3}{ }^{+}$primary ions over $200 \mu \mathrm{m} \times 200 \mu \mathrm{m}$ areas. Data analyses were performed on the negative ion spectra for each sample using the ION-TOF software.

\section{Results and discussion}

\subsection{DLS studies}

Z-average from a DLS measurement corresponds to the mean hydrodynamic diameter of the particles in a sample whilst PDI is a measure of the width of the particle size distribution.

125 PDI values of 0.0-0.1 point to a narrow monodisperse distribution, 0.1-0.4 indicate a moderate polydisperse distribution system, whereas values greater than 0.4 indicate a broadly polydisperse system. Generally, particles in a sample with mean sizes above $500 \mathrm{~nm}$ and PDIs greater than 0.5 are considered as "large or agglomerated", which may not be truly representative of the sample. This is because few large particles present in a sample usually dominate the light scattering 
130 signals in DLS analysis and give rise to large PDI values, which render the results unreliable. Nanoparticles with ZPs up to $|10 \mathrm{mV}|$ are considered "neutral" whereas those with ZPs greater than $|30 \mathrm{mV}|$ are considered "strongly charged" (Clogston and Patri, 2011).

Fig. 1 shows the size distributions by intensity of drug-free and drug-loaded (AmB or PAR) SLNs whilst Fig. 2 illustrates the z-averages and PDIs of the SLNs, before and after 135 exposure to the simulated fluids. Table 1 shows the ZP values of the SLN formulations in the various media.

\section{Table 1}

ZPs (mV) of drug-free and drug-loaded SLNs obtained from DLS.

\begin{tabular}{lllll}
\hline $\begin{array}{l}\text { SLN } \\
\text { formulation }\end{array}$ & Fresh sample & SGF-only & SIF-only & SGF+SIF \\
\hline Drug-free & $-67.6 \pm 1.7$ & $2.4 \pm 0.3$ & $-28.9 \pm 0.6$ & $-0.4 \pm 0.1$ \\
AmB & $-61.9 \pm 1.0$ & $1.1 \pm 0.6$ & $-31.2 \pm 1.2$ & $-3.0 \pm 0.1$ \\
& $-71.9 \pm 0.9$ & $4.9 \pm 1.1$ & $-35.7 \pm 0.5$ & $3.4 \pm 1.0$ \\
\hline
\end{tabular}

The SGF contained approximately $2.0 \mathrm{~g} / \mathrm{L}$ of $\mathrm{NaCl}$ and $2.917 \mathrm{~g} / \mathrm{L}$ of $\mathrm{HCl}$ as specified in the United States and European Pharmacopoeias. SGF is less complex as compared with in vivo gastric fluid. However, the dominant features of stomach fluid are its acidity and ionic content, which are replicated with the SGF. 
There was an approximately 3.8 -fold increase in the $\mathrm{z}$-average of the drug-free particles in SGF, which points to massive aggregation of the particles. The marked drop in the magnitude of $\mathrm{ZP}$, as well as the change in surface charge from negative to positive $(-67.6 \pm 1.7 \mathrm{mV}$ to $2.4 \pm$ $0.3 \mathrm{mV}$ ) can be attributed to the $\mathrm{H}^{+}$ions in SGF neutralising the negative charge density on the fresh SLNs. Furthermore, the reduction in ZP favours van der Waals type interparticle interactions, which result in particle aggregation.

The SIF contained approximately $0.616 \mathrm{~g} / \mathrm{L}$ of $\mathrm{NaOH}$ and $6.8 \mathrm{~g} / \mathrm{L}$ of $\mathrm{KH}_{2} \mathrm{PO}_{4}$. In SIFonly, there was a 1.8-fold increase in particle size and a 2.3-fold decrease in the ZP after exposure of the fresh particles to the medium. The PDI value indicates a relatively narrower size distribution width of the particles in SIF-only than in SGF-only and no aggregation of the SLNs 155 in the former, which is due to retention of adequate surface charge $(|28.9 \mathrm{mV}|)$ above the threshold attendant for the agglomeration of colloidal particles (|15 mV|) (Riddick, 1968).

The composition and volume of fluid along the GI tract, the motility of the GI tract, as well as the transit of dosage forms within it are highly variable. As a result, there is no perfect representative model for GI fluid. However, in the media exposure simulations described above, the SGF+SIF medium approximates the gastric emptying of contents into the small intestine, which is fairly consistent with that in the gut. Within the context of drug release and uptake of particles within the gut, surface chemistry and size changes of the particles are key determinants. Simulation of gastric emptying in respect of exposure to GI fluids was therefore a good measure of what might be happen in vivo. The z-average, PDI and ZP of the drug-free SLNs, as obtained in SGF+SIF were $517.0 \pm 113.0 \mathrm{~nm}, 0.54 \pm 0.22$ and $-0.4 \pm 0.1 \mathrm{mV}$, respectively. Although a very low ZP magnitude is suggestive of particle agglomeration and subsequent precipitation, the PDI and mean particle size are intermediate between the values obtained in SGF-only and SIF- 
only. This probably suggests that the SLN aggregates in the SGF-only were held loosely as floccules, which then became deaggregated on exposure to SIF. A high ZP value is essential for the long-term storage stability of colloidal formulations. After oral administration however, only a sufficient ZP magnitude is necessary to maintain electrostatic repulsion among the particles prior to their absorption. As a result, a low ZP of nanoparticles after contact with GI fluids is not crucial in the present pursuit if their final size is optimal for absorption.

A study by Shakweh et al. showed that uptake of neutral and negatively charged nano-

175 and microparticles by Peyer's patches in mice occurred to a greater extent as compared with that of positively charged particles (Shakweh et al., 2005). They observed a much larger number of particles with mean diameters of approximately 300 to $1000 \mathrm{~nm}$ in the patches than larger-sized particles. Again, particles with negative or close to neutral ZPs did not interact with the intestinal mucus gel layer due to electrostatic repulsion with the carboxylic $\left(\mathrm{COO}^{-}\right)$moiety of mucin, thus 180 allowing such particles to reach the follicle-associated epithelium and subsequently the M cells, via endocytosis. Positively charged particles on the other hand were found to interact strongly with the mucus layer as the latter is negatively charged, resulting in an impeded uptake (Shakweh et al., 2005).

The aforementioned results suggest that, in SGF+SIF, the drug-free particles retained their integrity as regards size and surface charge, which are critical features needed to augment absorption via endocytosis and subsequent lymphatic transport (Jani et al., 1989, 1994; Shakweh et al., 2005). The increase in size observed after exposure of the particles to the media may be a result of either aggregation or ingress of the dissolution media into the particles or both. The size distribution of the AmB SLNs (Fig. 1) in SGF-only was bimodal, and the two particle populations had mean sizes of $115.3 \mathrm{~nm}$ and $737.7 \mathrm{~nm}$. As with the drug-free particles, the AmB 
SLNs aggregated in SGF and after transfer from SGF into SIF, some deaggregation occurred. Prior to incubation, the AmB SLNs had z-average of $210.1 \pm 1.4 \mathrm{~nm}$, PDI of $0.22 \pm 0.01$ and ZP of $-61.9 \pm 1.0 \mathrm{mV}$.

There were no marked differences between the DLS data obtained for drug-free and AmB SLNs. Interestingly, both formulations showed an identical pattern of size change in the respective media, with the largest increase in size occurring in SGF-only, followed by SGF+SIF and then SIF-only.

The increase in the sizes of the particles in SGF-only and SGF+SIF can be attributed to aggregation while the comparatively less marked size increase in SIF-only could be the result of 200 influx of dissolution medium into the particles. The aggregation of the SLNs in SGF is likely to result in a reduced release of $\mathrm{AmB}$ from the particles due to a decrease in effective surface area. Consequently, aside from slow drug release from SLNs due to the hydrophobic nature of the lipid matrix, which retains most of the encapsulated drug within the matrix, particle aggregation in an acidic medium as observed above may further favour slow or no drug release in the 205 stomach. This attribute is desirable as the small intestine, with its high expression of Peyer's patches, is the probable site for uptake of the SLNs. Furthermore, the data obtained from our previous work in rats (Amekyeh et al., 2015) supports the assertion that AmB SLNs were taken up mostly from the small intestine.

The PAR SLNs showed a bimodal distribution in SIF-only with mean sizes of $156.1 \mathrm{~nm}$ and $596.5 \mathrm{~nm}$ (Fig. 1). The size of the PAR SLNs was highest in SGF-only, followed by SIFonly and then SGF+SIF. Generally, the increase in particle size after incubation in the media was lower for PAR SLNs as compared with the drug-free and AmB SLNs. 
From previous studies, it appears that either a decrease or a size retention of various SLN formulations was observed in SGF-only (Jain et al., 2012; Laserra et al., 2015; Zhang et al., 215 2013) while in SIF-only (Jain et al., 2012; Laserra et al., 2015; Roger et al., 2009; Yuan et al., 2013), either an increase or a decrease in size was observed. It is likely that the composition of SLNs contribute to their propensity to aggregate as a function of GI media. In the present work, a size increase or aggregation of both drug-free and AmB-loaded SLNs was observed in the simulated GI fluids and this was manifested more in SGF-only.

The hydrodynamic size obtained from DLS measurements can be affected by the salt concentration in a sample. This is due to the electrical double layer surrounding charged particles in an aqueous medium, which can be extended by the salts and result in an increase in size (Hackley and Clogston, 2011). In DLS, the intensity of scattered light is proportional to the square of particle diameter. Therefore, larger particles or clusters of smaller particles scatter light 225 more strongly than smaller particles do (Balog et al., 2015).

The marked increase in sizes of the SLNs observed in the present work could therefore be attributed to the few aggregated particles having approximate sizes of $5000 \mathrm{~nm}$, which can be seen in each of the size distribution profiles for the fresh SLNs (Fig. 1). These large particles can perturb the light scattering signals, mask the presence of the smaller particles and lead to biased and possibly inaccurate size measurements.

For samples containing significant differences in size populations such as the bimodal distributions of the AmB SLNs in SGF-only and the PAR SLNs in SIF-only, the calculated zaverages do not convey very accurate size information. Although the DLS software has been designed to correct this aberration in multimodal distributions, the results may not reflect true 
235 particle size distributions. As such, other particle sizing techniques can be used in addition to DLS in such instances (Lim et al., 2013).

Overall, ZP was highest for the fresh SLN samples (Table 1). This is because the dispersion medium used to prepare the SLNs was deionised water. Hence, the negative surface charge on the fresh particles was solely due to the anionic surfactant (sodium cholate) used for

240 formulating the SLNs. Incubating the SLNs in the simulated media resulted in a decrease in ZP, due the presence of electrolytes in the fluids. This is because $\mathrm{ZP}$ is dependent on the $\mathrm{pH}$ and conductivity of the suspending medium (Clogston and Patri, 2011). However, the decrease in ZP was more marked in SGF-containing media due to charge neutralisation by the $\mathrm{H}^{+}$ions present in SGF.

In SGF+SIF, the drug-loaded SLNs had mean sizes ranging from 362.8 to $513.2 \mathrm{~nm}$ and neutral ZPs ranging from -10 to $+10 \mathrm{mV}$. These properties favour the absorption of the SLNs via endocytosis by intestinal cells as well as via the Peyer's patches through lymphatic transport (Clogston and Patri, 2011; Shakweh et al., 2005). We may conclude that the SLNs exhibit ideal physical properties necessary for absorption, following oral delivery and transit from the 250 stomach to the small intestine.

\subsection{NTA studies}

In order to investigate the size variation of the SLNs in simulated GI fluids, free from the bias of DLS, the particle-by-particle approach for determining size using NTA was used. Fig. 3

255 shows the mean particle size of the drug-free and drug-loaded SLNs, before and after their incubation in the various media. There was a general increase in size after incubating the 
particles in the simulated media, except for the AmB SLNs in SGF+SIF and the PAR SLNs in all three media. The current results are suggestive of increase in particle size due to ingress of the media into the particles rather than of particle aggregation as indicative of the DLS data. The

260 largest increase recorded for the drug-free particles was $94.1 \mathrm{~nm}$ and that was observed in $\mathrm{SGF}+\mathrm{SIF}$.

For the AmB SLNs, there was an increase in size in SGF-only and SIF-only and a decrease in size in SGF+SIF. AmB is insoluble in water at $\mathrm{pH}$ 6-7 but soluble at $\mathrm{pH} 2$ or 11 due to its amphoteric nature. It appears that $\mathrm{SGF}+\mathrm{SIF}$, having an approximate $\mathrm{pH}$ of 1.5 , served as a better dissolution medium for AmB. In this regard, the diffusion of AmB from the particles resulted in a size decrease as opposed to the increase in size observed in the other media

For the PAR SLNs, there was a decrease in size in all the three media and the largest decrease was observed in SGF+SIF. PAR is a hydrophilic drug and slightly soluble in water. PAR is also weakly acidic and forms a salt in strong acids (SGF) or bases however, the solubility 270 of this salt depends on the ionic content of the dispersion medium. Since the dissolution of weakly acidic drugs is very minimal in strong acids, we observed identical size changes of the PAR SLNs in SGF-only and SIF-only (185.4 nm versus $182.7 \mathrm{~nm}$, respectively), possibly indicating similar solubility characteristics of the drug at the two pHs respectively. In this regard, ingress of dissolution media into the particles would be followed by dissolution and diffusion out 275 of the particles, resulting in the observed decrease in particle size.

Comparing DLS and NTA, the latter is time-consuming and requires more operational skills but it clearly has benefits over DLS. NTA allows for sample visualisation, gives an approximate concentration of the particles in a sample and produces size data based on the Brownian motion of individual particles. The presence of few large particles in a sample has 
280 little impact on the sizing accuracy of the NTA technique (Filipe et al., 2010). However, this is not the case in DLS, in which larger particles perturb and dominate the light scattering signals (Clogston and Patri, 2011). This could have been the reason for the observed differences in particle sizes from using the two techniques. In the present study, much larger particle sizes were obtained using DLS as compared with NTA although all the samples were similarly treated in 285 the various media. Notwithstanding, the two techniques complement each other.

In summary, contrasting patterns of size changes were observed for the drug-loaded SLNs in the various media. It appears that the size changes were dependent on the solubility of the incorporated drugs in the simulated fluids such that, ingress of the dissolution media into the SLNs caused some initial swelling of the particles. The dissolved drug was then free to diffuse 290 out of the matrix of the particles, the rate of which depends on the aqueous solubility of the drug. Therefore, with PAR being the more hydrophilic drug in the present study, the PAR SLNs manifested a larger decrease in size due to the faster diffusion rate of the drug from the SLN matrices into the aqueous media. Particles with sizes below $10 \mu \mathrm{m}$ are well taken up in the GI tract but uptake occurs more favourably when sizes are below $1000 \mathrm{~nm}$ (Jani et al., 1989;

295 Kreuter, 1991; Shakweh et al., 2005). In addition, the mesh-pore spacing of the intestinal mucosal barrier is 50-1800 nm (Lai et al., 2010; Primard et al., 2010).

The SLN formulations were found to be stable in the simulated GI fluids and are therefore deemed to be stable in vivo as well. The mean particle sizes and ZPs for all three formulations in the simulated media were also optimal for GI absorption meaning that the different particles would respond similarly with the GI mucus and epithelia. 


\subsection{ToF-SIMS analyses}

The negative and positive secondary ion spectra of the pure drugs, drug-free and drugloaded SLNs as well as the individual ingredients used for preparing the SLNs were subjected to ToF-SIMS analyses. However, only the negative ion spectra are presented as they provided data that were more meaningful as regards yields of the secondary ions of fragmented chemical components of the particles. In addition, the spectra for the individual non-drug components of the SLNs are not presented as these constituted the drug-free SLNs.

The ions representing the diagnostic peaks used to identify $\mathrm{AmB}\left(\mathrm{C}_{47} \mathrm{H}_{73} \mathrm{NO}_{17}, 923.49\right.$ $\mathrm{g} / \mathrm{mol}$ ) on the SLNs were $\left[\mathrm{C}_{20} \mathrm{H}_{27} \mathrm{O}_{2}\right]^{-}$at $m / z$ 299.20, $\left[\mathrm{C}_{20} \mathrm{H}_{29} \mathrm{O}_{2}\right]^{-}$at $m / z, 301.22$ and $\left[\mathrm{C}_{17} \mathrm{H}_{21} \mathrm{NO}_{4}\right]^{-}$ at $m / z 303.23$.

Comparing pure AmB with AmB SLNs, the peak intensities of the diagnostic ions (Fig. 4(a)) indicated that the drug was present only in small quantities on the surface of the freshly prepared SLNs. As there was no drug loaded in the drug-free nanoparticles, the peaks corresponding to the diagnostic ions in the spectrum for the drug-free SLNs are due to the other ingredients used in preparing the nanoparticles. As a result, the peaks at $m / z, 299.20,301.22$ and 303.23 in the spectrum for the AmB SLNs could be due to the excipients in the formulation, especially since the peak intensities are higher for the drug-free particles. These factors point to a drug-enriched SLN core and are a reflection of the high encapsulation efficiency of $91.2 \pm 3.0 \%$ obtained for the AmB SLNs in our previous study (Amekyeh et al., 2015).

In Fig. 5(a), the relative intensities of AmB on the surfaces of the fresh SLNs and their after exposure to the media are compared. It can be observed that higher drug counts were detected on the particles after exposure of the SLNs to the GI media in the following order: 
SGF+SIF > SIF-only > SGF-only. This trend is the reverse order of the particle size of the AmB

325 SLNs in the respective media, as obtained from the NTA data. The higher intensities of the reference peaks for the media-exposed SLNs compared to the fresh sample points to the diffusion of AmB towards the surfaces of the SLNs. It is apparent that this diffusion to the surface is most effective in SGF+SIF and least so in SGF-only. Furthermore, this observation is in concert with the assertion that the diffusion of AmB from the SLN matrices is preceded by ingress of the media into the nanoparticles and dissolution of the payload, the speed of which may result in shrinkage of particles to various extents.

The diagnostic peak for PAR $\left(\mathrm{C}_{8} \mathrm{H}_{9} \mathrm{NO}_{2}, 151.163 \mathrm{~g} / \mathrm{mol}\right)$ is at $\mathrm{m} / \mathrm{z}$ of 107.05 , representing the $\left[\mathrm{C}_{7} \mathrm{H}_{7} \mathrm{O}\right]^{-}$ion. The peak at $m / z 107.05$ (Fig. 4(b)) in the spectrum for the drug-free SLNs is attributable to the excipients used in the formulation. The intensity of the diagnostic peak was higher on the drug-loaded SLNs than on the drug-free particles, which indicates that the peak intensity observed on the SLNs is a combination of that for the drug itself as well as the excipients. This observation is the reverse of that detected in Fig. 4(a) for AmB. The results therefore indicate that there is a little amount of PAR on the surface of the fresh PAR SLNs, unlike the AmB SLNs, which had a negligible amount of AmB on the SLNs.

In Fig. 5(b), the spectra show a much intense PAR count on the fresh PAR nanoparticles in comparison to those incubated in the media. The PAR SLNs showed a relatively low encapsulation efficiency of $60.7 \pm 0.26 \%$ (Amekyeh et al., 2015).

PAR is hydrolysed in acidic solutions (SGF) to produce 4-aminophenol and acetic acid; however, these products have different masses and would therefore not contribute to the signal intensity at $\mathrm{m} / \mathrm{z}$ 107.05. This would manifest as a low PAR count on the particles in SGF-only as 
compared with the fresh particles. Furthermore, PAR is very soluble at the $\mathrm{pH}$ of SIF, which favours drug release and dissolution in the medium resulting in an equally low drug count on the particles. As with the AmB SLNs, the highest drug intensity was detected on the PAR SLNs after their incubation in SGF+SIF, which indicates the latter as the best dissolution medium for both drugs. This means that there is a higher rate of drug diffusion out of the particles in SGF+SIF, which explains why the two SLNs had the smallest mean particle size in that medium (Fig. 3).

The mean particle size (based on the NTA data) of the PAR SLNs after exposure to the media decreased in the order: SGF-only $>$ SIF-only $>$ SGF+SIF, and drug intensity on the SLNs was SIF-only < SGF-only < SGF+SIF. From Fig. 3, the mean sizes of the PAR nanoparticles in

355 SGF-only and SIF-only are comparable $(185.4 \pm 53.9 \mathrm{~nm}$ and $182.7 \pm 27.2 \mathrm{~nm}$, respectively). Also, in Fig. 5(b), the intensity of the diagnostic peak is similar in the two media albeit slightly lesser in SIF-only, unequivocally signifying that the ultimate size of the particles is inversely related to the amount of drug released from the particles into the medium.

\section{Conclusions}

The data obtained indicate that after placing the SLNs in the simulated GI media, there was ingress of the fluids into the SLNs followed by diffusion of the dissolved drug. The rate of the diffusion however depended on the solubility of the loaded-drug in the particular medium. There was an increase in the size (NTA-based) of the drug-free SLNs in all the three media since

365 the main mass transfer was the diffusion of the media into the SLNs and no drug diffusion out of the particles. The study showed NTA to be a more suitable technique than DLS for analysing the stability of the SLNs in the various media. The data obtained indicate that the SLNs after oral 
ingestion may aggregate in the stomach. However, on reaching the small intestine, both the size and surface charge of the particles would be optimal for absorption. The mass spectra obtained from the ToF-SIMS analyses indicated that drug loading into the SLNs possibly followed the core-shell model and that the AmB SLNs may have a more drug-enriched core than the PAR SLNs do. The AmB SLNs are therefore a very suitable carrier of AmB for oral delivery. Furthermore, the stability of the SLN formulations in the simulated GI media indicated their suitability for oral delivery.

\section{Acknowledgements}

We also thank the Laboratory of Biophysics and Surface Analysis (LBSA) and the Nottingham Nanotechnology and Nanoscience Centre, both at the University of Nottingham (UK campus) for the facilities and support provided throughout the study. 
FIGURES
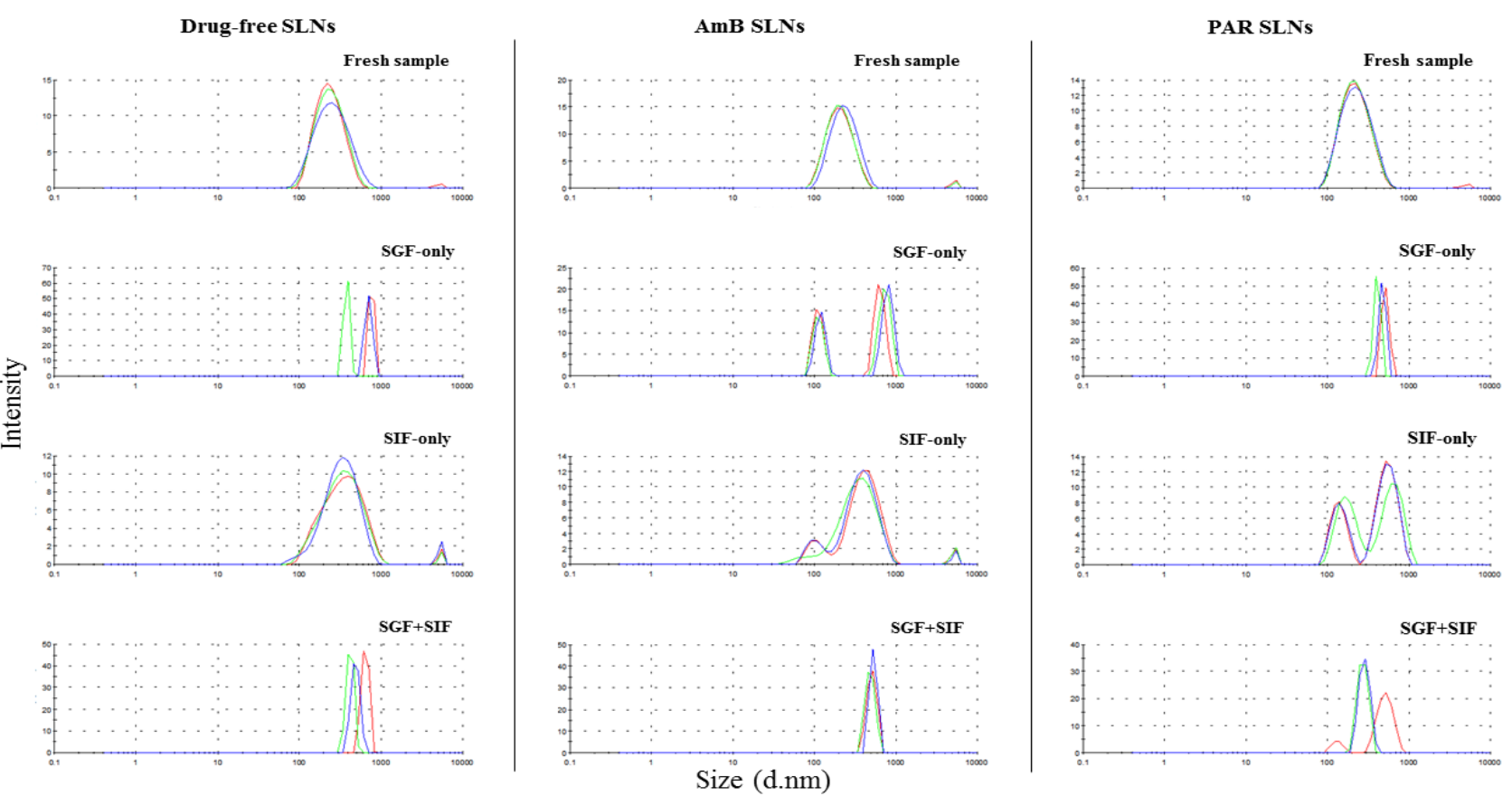

Figure 1 Size distribution by intensity of drug-free and drug-loaded SLNs, before and after their exposure to simulated GI fluids. The analyses were conducted using DLS. Triplicate measurements were performed for each sample.

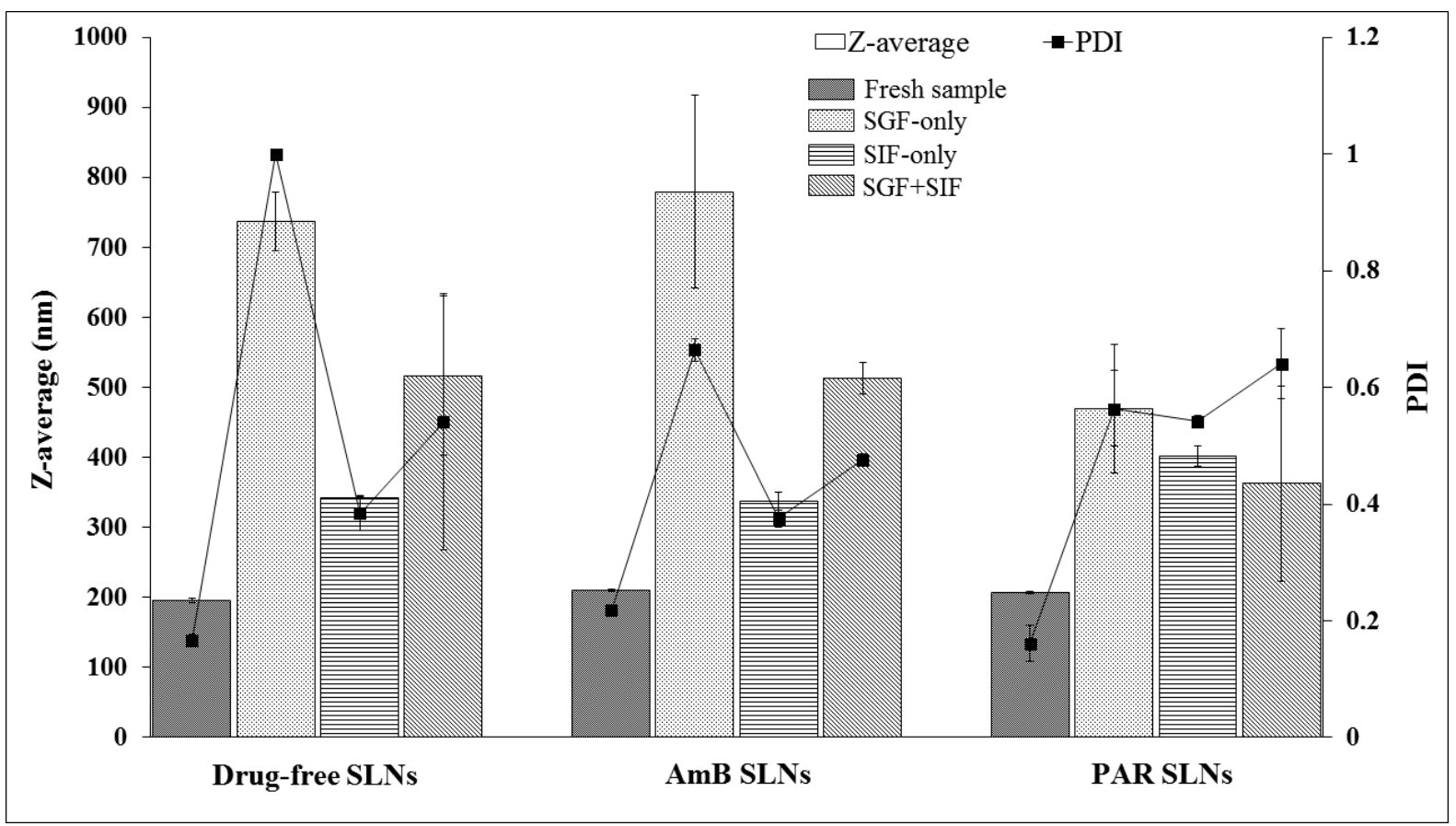

Figure 2 Z-averages and PDIs of drug-free and drug-loaded SLNs, before and after their exposure to simulated GI fluids. The analyses were conducted using DLS. Triplicate measurements were performed for each sample. Data have been presented as mean \pm standard deviation. 


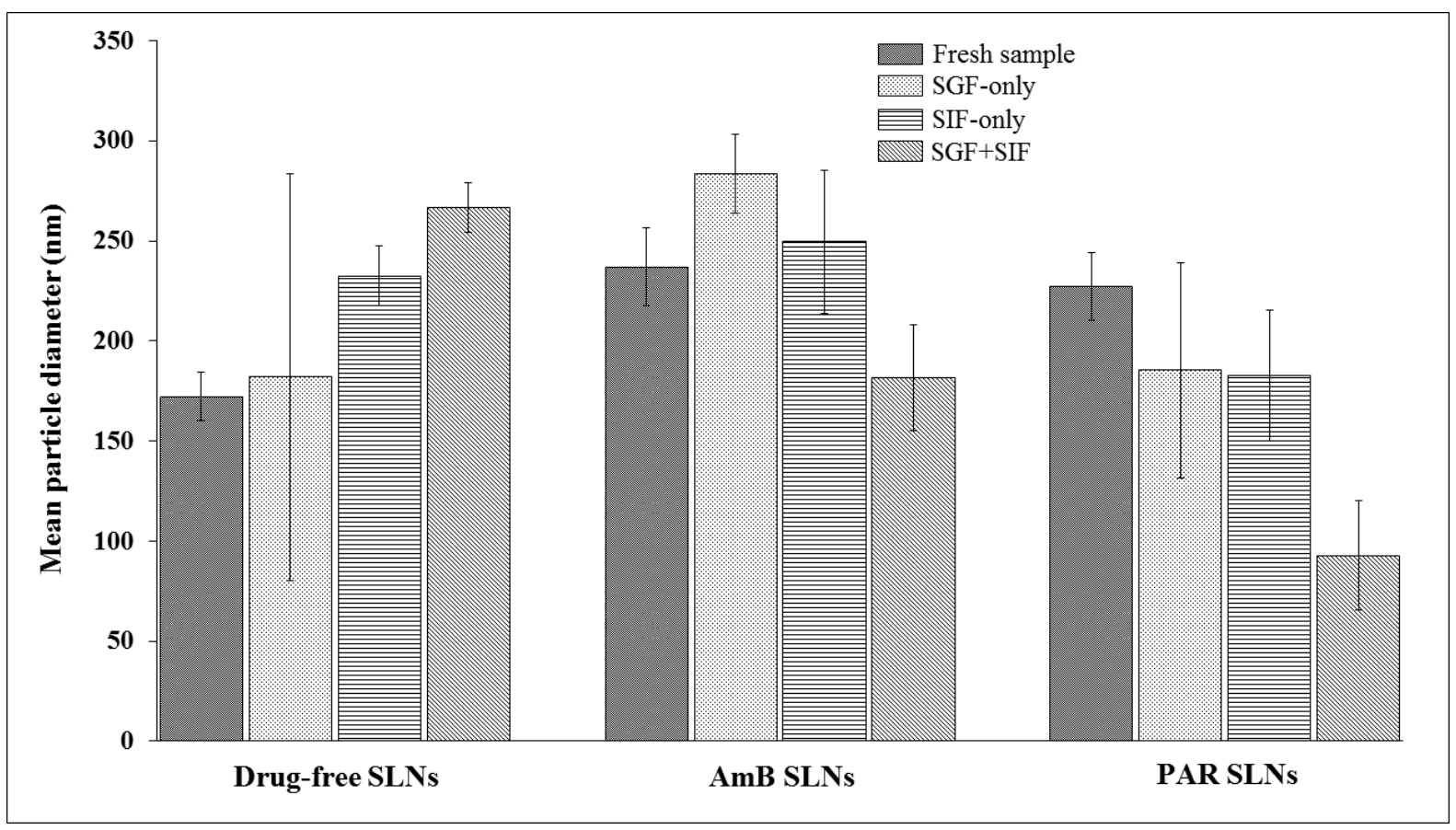

Figure 3 Mean particle diameter of drug-free and drug-loaded SLNs, before and after their exposure to simulated GI fluids. Measurements were done using NTA. Triplicate measurements were performed for each sample. Data have been presented as mean \pm standard deviation.

(a) $\mathrm{AmB}$

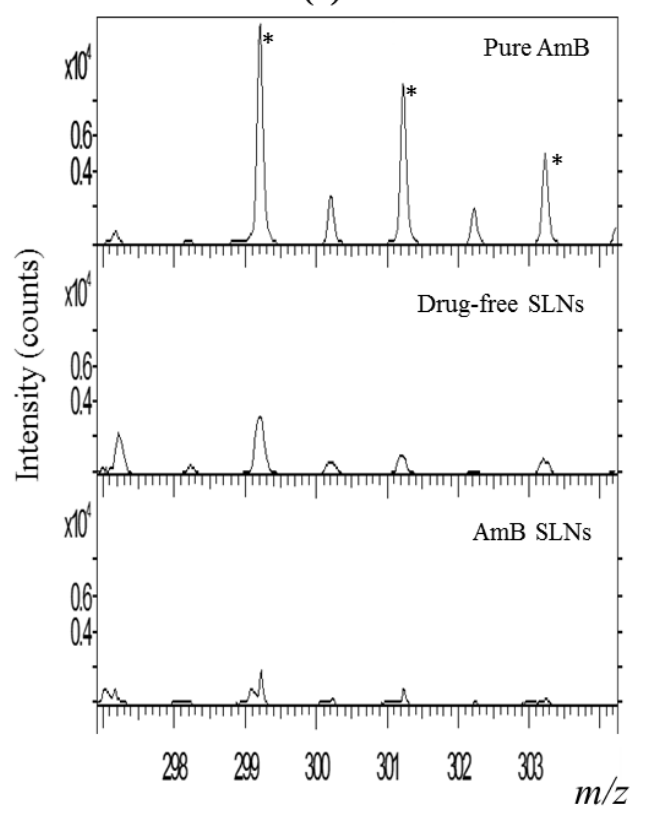

(b) PAR

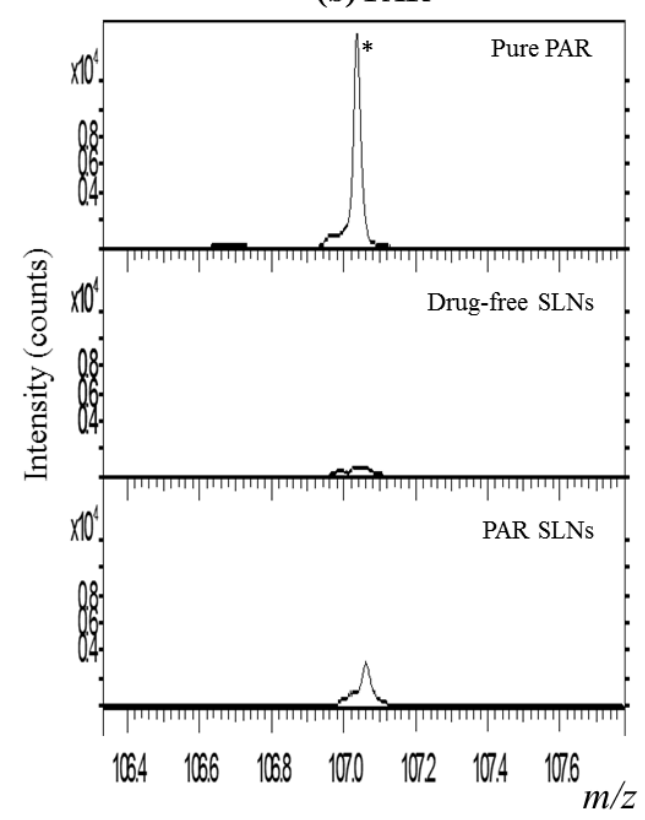

Figure 4 Negative ion ToF-SIMS spectra showing pure drugs [(a) AmB or (b) PAR], drug-free SLNs and the respective drug-loaded SLNs. [* ${ }^{*}$ diagnostic peaks]. 
(a) AmB SLNs

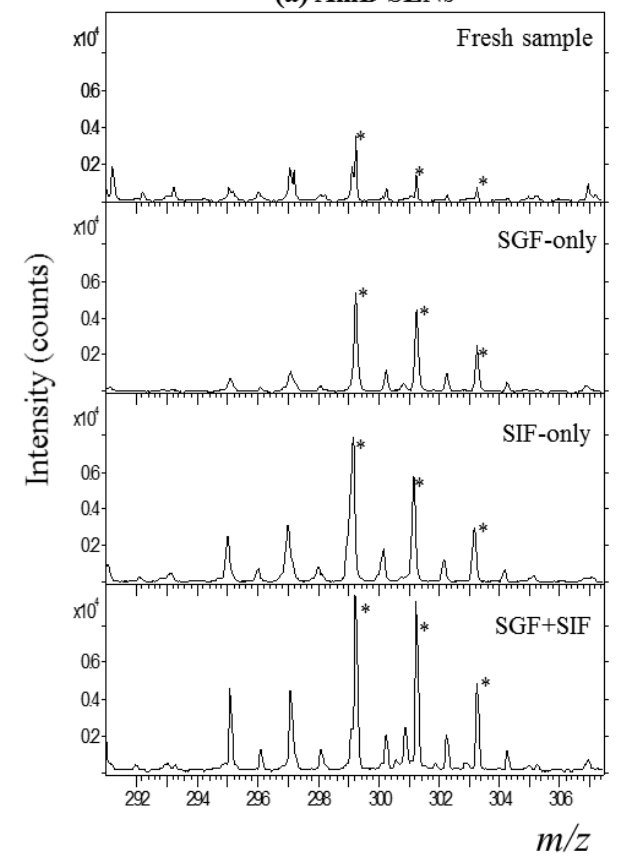

(b) PAR SLNs

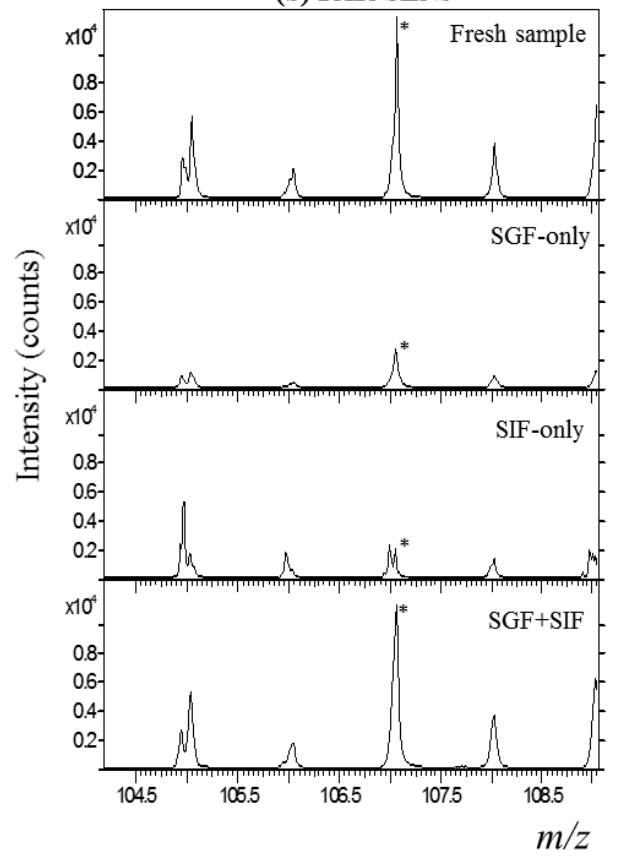

Figure 5 Negative ion ToF-SIMS spectra showing the drugs [(a) AmB or (b) PAR] on the surface of the respective fresh SLNs and after their incubation in simulated GI fluids. The asterisks represent the diagnostic peaks. 


\section{References}

Amekyeh, H., Billa, N., Yuen, K.H., Chin, S.L.S., 2015. A gastrointestinal transit study on amphotericin B-loaded solid lipid nanoparticles in rats. AAPS PharmSciTech 16, 871877. doi:http://dx.doi.org/10.1208/s12249-014-0279-4.

Balog, S., Rodriguez-Lorenzo, L., Monnier, C.A., Obiols-Rabasa, M., Rothen-Rutishauser, B., Schurtenberger, P., Petri-Fink, A., 2015. Characterizing nanoparticles in complex biological media and physiological fluids with depolarized dynamic light scattering. Nanoscale 7, 5991-5997. doi:http://dx.doi.org/10.1039/C4NR06538G.

Clogston, J.D., Patri, A.K., 2011. Zeta potential measurement, in: McNeil, S.E. (Ed.), Methods in molecular biology: Characterization of nanoparticles intended for drug delivery. Humana Press, New York, pp. 63-70. doi:http://dx.doi.org/10.1007/978-160327-198-1_6.

Damgé, C., Michel, C., Aprahamian, M., Couvreur, P., Devissaguet, J.P., 1990. Nanocapsules as carriers for oral peptide delivery. J. Control. Release 13, 233-239. doi:http://dx.doi.org/10.1016/0168-3659(90)90013-J.

Filipe, V., Hawe, A., Jiskoot, W., 2010. Critical evaluation of nanoparticle tracking analysis (NTA) by NanoSight for the measurement of nanoparticles and protein aggregates. Pharm. Res. 27, 796-810. doi:http://dx.doi.org/10.1007/s11095-010-0073-2.

Hackley, V.A., Clogston, J.D., 2011. Measuring the hydrodynamic size of nanoparticles in aqueous media using batch-mode dynamic light scattering, in: McNeil, S.E. (Ed.), Methods in molecular biology: Characterization of nanoparticles intended for drug delivery. Humana Press, New York, pp. 36-52. doi:http://dx.doi.org/10.1007/978-160327-198-1_4.

Jain, S., Valvi, P.U., Swarnakar, N.K., Thanki, K., 2012. Gelatin coated hybrid lipid nanoparticles for oral delivery of amphotericin B. Mol. Pharm. 9, 2542-2553. doi:http://dx.doi.org/10.1021/mp300320d.

Jani, P., Halbert, G.W., Langridge, J., Florence, A.T., 1989. The uptake and translocation of latex nanospheres and microspheres after oral administration to rats. J. Pharm. Pharmacol. 41, 809-812. doi:http://dx.doi.org/10.1111/j.2042-7158.1989.tb06377.x. 
Jani, P., Halbert, G.W., Langridge, J., Florence, A.T., 1990. Nanoparticle uptake by the rat gastrointestinal mucosa: quantitation and particle size dependency. J. Pharm. Pharmacol. 42, 821-826. doi:http://dx.doi.org/10.1111/j.2042-7158.1990.tb07033.x.

Jani, P.U., McCarthy, D.E., Florence, A.T., 1994. Titanium dioxide (rutile) particle uptake from the rat GI tract and translocation to systemic organs after oral administration. Int. J. Pharm. 105, 157-168. doi:http://dx.doi.org/10.1016/0378-5173(94)90461-8.

Kreuter, J., 1991. Peroral administration of nanoparticles. Adv. Drug Deliv. Rev. 7, 71-86. doi:http://dx.doi.org/10.1016/0169-409X(91)90048-H.

Lai, S.K., Wang, Y.Y., Hida, K., Cone, R., Hanes, J., 2010. Nanoparticles reveal that human cervicovaginal mucus is riddled with pores larger than viruses. Proc. Natl. Acad. Sci. USA 107, 598-603. doi:http://dx.doi.org/10.1073/pnas.0911748107.

Laserra, S., Basit, A., Sozio, P., Marinelli, L., Fornasari, E ., Cacciatore, I., Ciulla, M., Türkez, H., Geyikoglu, F., Di Stefano, A., 2015. Solid lipid nanoparticles loaded with lipoyl-memantine codrug: Preparation and characterization. Int. J. Pharm. 485, 183-191. doi:http://dx.doi.org/10.1016/j.ijpharm.2015.03.001.

Lim, J., Yeap, S.P., Che, H.X., Low, S.C., 2013. Characterization of magnetic nanoparticle by dynamic light scattering. Nanoscale Res. Lett. 8, 381. doi:http://dx.doi.org/10.1186/1556-276X-8-381.

Müller, R.H., Ruhl, D., Runge, S.A., 1996. Biodegradation of solid lipid nanoparticles as a function of lipase incubation time. Int. J. Pharm. 144, 115-121. doi: http://dx.doi.org/10.1016/S0378-5173(96)04731-X.

Muthu, M.S., Feng, S.S., 2009. Pharmaceutical stability aspects of nanomedicines. Nanomedicine 4, 857-860. doi:http://dx.doi.org/10.2217/nnm.09.75.

Olbrich, C., Kayser, O., Müller, R.H., 2002a. Enzymatic degradation of Dynasan 114 SLN effect of surfactants and particle size. J. Nanopart. Res. 4, 121-129. doi:http://dx.doi.org/10.1023/A:1020159331420.

Olbrich, C., Kayser, O., Müller, R.H., 2002b. Lipase degradation of Dynasan 114 and 116 solid lipid nanoparticles (SLN)-effect of surfactants, storage time and crystallinity. Int. J. Pharm. 237, 119-128. doi:http://dx.doi.org/10.1016/S0378-5173(02)00035-2. 
Pouton, C.W., 2000. Lipid formulations for oral administration of drugs: nonemulsifying, self-emulsifying and 'self-microemulsifying' drug delivery systems. Eur. J. Pharm. Sci. 11, S93-S98. doi:http://dx.doi.org/10.1016/S0928-0987(00)00167-6.

Primard, C., Rochereau, N., Luciani, E., Genin, C., Delair, T., Paul, S., Verrier, B., 2010. Traffic of poly (lactic-acid) nanoparticulate vaccine vehicle from intestinal mucus to sub-epithelial immune competent cells. Biomaterials 31, 6060-6068. doi:http://dx.doi.org/10.1016/j.biomaterials.2010.04.021.

Riddick, T.M., 1968. Control of colloid stability through zeta potential, Livingston Publishing Company, Pennsylvania.

Roger, E., Lagarce, F., Benoit, J.P., 2009. The gastrointestinal stability of lipid nanocapsules. Int. J. Pharm. 379, 260-265. doi:http://dx.doi.org/10.1016/j.ijpharm.2009.05.069.

Shakweh, M., Besnard, M., Nicolas, V., Fattal, E., 2005. Poly (lactide-co-glycolide) particles of different physicochemical properties and their uptake by Peyer's patches in mice. Eur. J. Pharm. Biopharm. 61, 1-13. doi:http://dx.doi.org/10.1016/j.ejpb.2005.04.006.

Subramanian, N., Ghosal, S.K., 2004. Enhancement of gastrointestinal absorption of poorly water soluble drugs via lipid based systems. Indian J. Exp. Biol. 42, 1056-1065.

Tan, C.S.W., Billa, N., Roberts, C.J., Scurr, D.J., 2014. Properties of an oral nanoformulation of a molecularly dispersed amphotericin B comprising a composite matrix of theobroma oil and bee's wax. Nanomaterials 4, 905-916. doi:http://dx.doi.org/10.3390/nano4040905.

Tan, S.W., Billa, N., Roberts, C.R., Burley, J.C., 2010. Surfactant effects on the physical characteristics of Amphotericin B-containing nanostructured lipid carriers. Colloid. Surface. A 372, 73-79. doi:http://dx.doi.org/10.1016/j.colsurfa.2010.09.030.

Wasan, E.K., Bartlett, K., Gershkovich, P., Sivak, O., Banno, B., Wong, Z., Gagnon, J., Gates, B., Leon, C.G., Wasan, K.M., 2009. Development and characterization of oral lipid-based amphotericin B formulations with enhanced drug solubility, stability and antifungal activity in rats infected with Aspergillus fumigatus or Candida albicans. Int. J. Pharm. 372, 76-84. doi:http://dx.doi.org/10.1016/j.ijpharm.2009.01.003. 
Yuan, H., Chen, C.Y., Chai, G., Du, Y.Z., Hu, F.Q., 2013. Improved transport and absorption through gastrointestinal tract by pegylated solid lipid nanoparticles. Mol. Pharm. 10, 1865-1873. doi:http://dx.doi.org/10.1021/mp300649z.

Zhang, C., Gu, C., Peng, F., Liu, W., Wan, J., Xu, H., Lam, C.W., Yang, X., 2013. Preparation and optimization of triptolide-loaded solid lipid nanoparticles for oral delivery with reduced gastric irritation. Molecules 18, 13340-13356. doi:http://dx.doi.org/10.3390/molecules181113340. 